\title{
HST SPECTROSCOPY OF THE ORION NEBULA
}

\author{
R.H. RUBIN \\ NASA/Ames \\ G.J. FERLAND \\ University of Kentucky \\ R.J. DUFOUR, D.K. WALTER, C.R. O'DELL \\ Rice University \\ J.A. BALDWIN \\ CTIO \\ J.J. HESTER \\ Arizona State University \\ AND \\ P.G. MARTIN \\ University of Toronto
}

\begin{abstract}
.
From our recent Faint Object Spectrograph (FOS) observations of the Orion Nebula obtained with the Hubble Space Telescope, we present preliminary results that address the nitrogen abundance. The list of detected lines and their identifications included the first measurement of the $\mathrm{N}$ II] $2142 \AA$ line in an $\mathrm{H}$ II region. This measurement in conjunction with [O II] $2471 \AA$ permits a new assessment of the important N/O ratio in Orion. Unfortunately, the measurements of the N III] 1747-54 $\AA$ multiplet and the O III] 1660-66 $\AA$ multiplet have poor signal-to-noise, precluding another independent derivation of $\mathrm{N} / \mathrm{O}$.
\end{abstract}

\section{Observations}

Several bright regions at varying distances from $\theta^{1}$ Ori $\mathrm{C}$ were observed during 1993 August with HST/FOS at 5-15 $\AA$ resolution. For three positions, we have total wavelength coverage from 1650-6800 $\AA$. Additionally, 
at the three positions, we observed at $3250<\lambda<6800 \AA$ through the upper member of the $1.0^{\prime \prime}$ pair of apertures (beam switching). The second member of the paired square aperture provided us with a position located $3^{\prime \prime}$ from each of our primary positions with little added exposure time. This allows us to investigate small-scale variations in ionization and physical conditions in the same part of the nebula.

For the purpose of providing a preliminary list of spectral lines measured, we derive an average of the spectra taken in the three lower apertures at our positions 1,2 , and $3-29^{\prime \prime}, 19^{\prime \prime}$, and $42^{\prime \prime}$ from $\theta^{1}$ Ori C. We have presented elsewhere a table listing measurements from this composite spectrum - with the measured wavelength, the vacuum rest wavelength and preliminary line identification, our measured flux in the $1^{\prime \prime} \times 1^{\prime \prime}$ aperture $\left(\mathrm{erg} \mathrm{cm}{ }^{-2} \mathrm{~s}^{-1}\right)$, the equivalent width $(\AA)$, and the FWHM $(\AA)$ (Rubin et al. 1994). Because this FOS observing configuration (the $1.0^{\prime \prime}$ pair of apertures) did not have proper calibration observations made until July 1994, the analysis and results presented here are subject to revision. Our Cycle 3 observations included a GHRS spectrum with the large science aperture at Position 1. This G270M exposure covered the region $2310-2360 \AA$ and provided the first detection of Si II] 2335.3 $\AA$ emission in an HII region (Walter et al. 1994).

\section{Discussion}

We detected the N II] $2142 \AA$ line(s) in the spectra at position 3 low only. The measurement of this line in Orion is the first in an $\mathrm{H}$ II region. Previously it has been seen in RR Tel (Penston et al. 1983), nova CrA 1981 (Williams et al. 1985), and the $\eta$ Car S condensation (Davidson et al. 1986). There are two lines that arise from ${ }^{5} S_{2}$ with lower levels ${ }^{3} P_{2}(2143.45 \AA)$ and ${ }^{3} P_{1}(2139.68 \AA)$ - in sum referred to as $2142 \AA$. The red component is expected to be more than twice as strong as the blue one. Preliminary analysis indicates that position 3 low has the highest $T_{e}$ as measured by the combined [ $\mathrm{N} \mathrm{II}]$ and $[\mathrm{O} \mathrm{III}]$ temperatures, which may be significant in explaining why the $\mathrm{N} \mathrm{II}] 2142 \AA$ line is strongest at this position. Further analysis of $T_{e}$ and temperature fluctuations in the $\mathrm{N}^{+}$zone using this line in conjunction with the 5755 and $6584 \AA$ lines, arising at the immediate lower levels, is underway. The measurement of N II] $2142 \AA$ in conjunction with [O II] $2471 \AA$ provides another measurement of the $\mathrm{N}^{+} / \mathrm{O}^{+}$abundance ratio that is less sensitive to uncertainties in knowledge of the $T_{e}$ value/distribution and to uncertainty in differential extinction compared with the more traditional optical method (see below).

Here we simply take the approach of retrofitting our two independent models of Orion (Baldwin et al. 1991; Rubin et al. 1991) to predict the 
N II] 2140-43 $\AA$ line and compare with the preliminary fluxes at position 3 low. For our retrofits at this time, in order to narrow the differences in input parameters, we arbitrarily used the Rubin et al. ionizing spectrum for $\theta^{1}$ Ori $\mathrm{C}$ and abundance set (whether meritorious or not). We use the same updated effective collision strengths and A-values to solve the $\mathrm{N}^{+}$energy level populations. Calamai \& Johnson (1991) have laboratory A-values for the $2140-43 \AA$ line. Recently calculated collision strengths affecting all 6 lowest-lying levels are from Stafford et al. (1994), although there is another contemporaneous set by Lennon \& Burke (1994) that has somewhat different values. For our separate models, we then prorate input nitrogen abundance to force the predicted line ratio to match the extinction-corrected observed N II $] 2142 /[\mathrm{O} \mathrm{II}] 2471$ ratio, yielding $(\mathrm{N} / \mathrm{O})_{u v}$. The same is done for the $[\mathrm{N} \mathrm{II}] 6584 /[\mathrm{O} \mathrm{II}] 3727$ ratio, which provides $(\mathrm{N} / \mathrm{O})_{\text {opt }}$ and the observed $[\mathrm{N} \mathrm{III}] 57 \mu \mathrm{m} /[\mathrm{O} \mathrm{III}] 52 \mu \mathrm{m}$ ratio, yielding $(\mathrm{N} / \mathrm{O})_{i r}$. Extinction is corrected for by using $\mathrm{A}(\lambda)$ (magn.) $=2.5 \mathrm{C}(\mathrm{H} \beta)[1+\mathrm{f}(\lambda)]$, where $\mathrm{C}(\mathrm{H} \beta)=0.64$ for our Orion position and $f(\lambda)$ is from Bohlin \& Savage (1981) in the UV and from Costero \& Peimbert (1970) in the optical. The effect of differential extinction is to increase the observed ratio $\mathrm{I}(2142) / \mathrm{I}(2471)$ by a factor of 1.20 , which is less than for $\mathrm{I}(3727) / \mathrm{I}(6584)$. For the $(\mathrm{N} / \mathrm{O})_{i r}$ determinations, we match to $\mathrm{I}(57) / \mathrm{I}(52)=0.13$ (Rubin et al. 1991) at a position which might be typical to our HST locations.

The retrofit of the Baldwin et al. model gives $(\mathrm{N} / \mathrm{O})_{u v}=0.109$, $(\mathrm{N} / \mathrm{O})_{o p t}=0.0840$, and $(\mathrm{N} / \mathrm{O})_{i r}=0.213$, while the retrofit of the Rubin et al. model gives respectively, $0.0843,0.128$, and 0.176 . There is much that remains to be done, awaiting final calibration of our FOS spectra, in terms of reconciling these spectral region differences and model differences. This preliminary analysis is to demonstrate the expanded opportunity HST data permit in regards to just one important "unsolved problem" - deriving $\mathrm{N} / \mathrm{O}$.

\section{Acknowledgements}

We thank Patrick Harrington for information regarding his measurements of N II] $2140-43 \AA$ in a FOS spectrum of a planetary nebula. RHR thanks Scott McNealy for providing a Sun workstation. We acknowledge NASA/Ames Rice Univ. and NASA/Ames - Univ. of Kentucky interchanges NCC2-5008 and NCC2-5028 as well as G0.4385 from STScI.

\section{References}

Baldwin, J.A., Ferland, G.J., Martin, P.G., Corbin, M.R., Cota, S.A., Peterson, B.M., \& Slettebak, A. 1991, ApJ, 374, 580

Bohlin, R.C., \& Savage, B.D. 1981, ApJ, 249, 109 
Calamai, A.G., \& Johnson, C.E. 1991, Phys Rev A, 44, 218

Costero, R., \& Peimbert, M. 1970, Bol. Obs. Ton. y Tacu., 5, 229

Davidson, K., Dufour, R.J., Walborn, N.R., \& Gull, T.R. 1986, ApJ, 305, 867

Lennon, D.J. \& Burke, V.M. 1994, A\&AS, 103, 273

Penston, M.V., et al. 1983, MNRAS, 202, 833

Rubin, R.H., Simpson, J.P., Haas, M.R., \& Erickson, E.F. 1991, ApJ, 374, 564

Rubin, R.H., et al. 1994, in The Analysis of Emission Lines, Proc. STScI Symp. (May 1994), Eds. R.E. Williams \& M. Livio

Stafford, R.P., Bell, K.L., Hibbert, A., \& Wijesundera, W.P. 1994, MNRAS, 268, 816

Walter, D.W., et al. 1994, in The Analysis of Emission Lines, Proc. STScI Symp. (May 1994), Eds. R.E. Williams \& M. Livio

Williams, R.E., et al. 1985, MNRAS, 212, 753 